\title{
What is New in the Clinical Assessment and Treatment of the Infertile Male
}

\author{
Sandro C. Esteves ${ }^{*}, 1$ and Ashok Agarwal ${ }^{2}$ \\ ${ }^{I}$ ANDROFERT, Andrology and Human Reproduction Clinic, Campinas, São Paulo, Brazil \\ ${ }^{2}$ Center for Reproductive Medicine, Cleveland Clinic, Cleveland, Ohio, USA
}

\begin{abstract}
Male infertility is a fast-evolving area. Recently published evidence has challenged several established concepts and added to the knowledge in the field. Traditionally, the clinical assessment of the subfertile male includes a history and physical examination, hormonal evaluation and semen analyses. Regarding the latter, the World Health Organization has established new reference values that in general include lower limits than those of previous editions. Specialized sperm function assays, such as sperm DNA integrity and measurements of oxidative stress, are now clinically available and may be integrated to the conventional semen analysis to provide a more comprehensive evaluation of a man's fertility status. Also, molecular biology genetic testing involving the Y-chromosome can correctly identify oligozoospermic and azoospermic men misdiagnosed as having idiopathic infertility and be of prognostic value for sperm retrieval in non-obstructive azoospermia. In the field of treatment, emerging evidence suggests that antioxidant therapy along with life-style modifications improve the male reproductive health. Microsurgery has increased the success rates for both varicocele treatment and sperm retrieval for assisted conception. Microsurgical treatment of clinical varicoceles may optimize reproductive outcome of couples undergoing intracytoplasmic sperm injection or microsurgical testicular sperm extraction. Men with non-obstructive azoospermia, elevated follicle-stimulating hormone levels and small testes may no longer be considered sterile. Modern retrieval techniques are used to collect testicular sperm that can be used to produce a healthy biological offspring via assisted conception. This article reviews what is new in the clinical assessment and treatment of the infertile male, including the implications of this increased knowledge in the management of the infertile couple and assisted reproduction technology outcomes.
\end{abstract}

Keywords: Male infertility, diagnosis, treatment, microsurgery, azoospermia, varicocele, assisted reproduction technology, sperm function tests.

\section{INTRODUCTION}

Infertility complaint is common in the urologic office. Approximately $8 \%$ of men in reproductive age seek medical assistance for fertility-related problems. Of these, $1-10 \%$ carries conditions that compromise the reproductive potential, and varicocele accounts for $35 \%$ of the cases [1]. Most infertility conditions are treatable, but in few cases they are associated with life-threatening diseases such as testicular, brain or spinal cord cancers, genitourinary malformations, genetic syndromes or systemic diseases. Therefore, comprehensive male workup should be an integral step in the evaluation of the infertile couple.

Recently published studies have added new evidence to this fast-evolving area. Novel diagnostic tools using molecular biology and DNA integrity or oxidative stress assays allow the clinician to better diagnose a significant proportion of men previously classified as having infertility of unknown etiology. Emerging evidence suggests that life-style and environmental conditions are of utmost importance in male fertility and subfertility. There are new opportunities for men with azoospermia or clinical varicoceles requiring assisted conception. Microsurgery has increased success rates in both

*Address correspondence to this author at the ANDROFERT, Av. Dr. Heitor Penteado, 1464, 13075-460, Campinas, São Paulo, Brazil; Tel: +55 19 3295-8877; Fax: +55 19 3294-6992; E-mail: s.esteves@androfert.com.br reconstruction of the reproductive tract and retrieval of spermatozoa for assisted conception. The present article reviews the current concepts in the clinical assessment and treatment of the infertile male.

\section{WHAT IS NEW IN THE CLINICAL ASSESSMENT}

\section{World Health Organization Reference Values for Human Semen Characteristics}

Semen analysis is of great value on the male initial investigation and its results are often taken as a surrogate measure of male fecundity and pregnancy risk. It provides information on the functional status of the germ epithelium, epididymides and accessory sex glands. Reference ranges for semen parameters from fertile populations may provide data from which prognosis of fertility or diagnosis of infertility can be extrapolated. Nonetheless, the prognostic value of semen components such as sperm number, motility and morphology, as surrogate markers of male fertility, is confounded in several ways; the fertility potential of a man is influenced by sexual activity, the function of accessory sex glands and other, defined as well as yet unrecognized, conditions. Routine semen analysis itself has its own limitations, and does not account for putative sperm dysfunctions such as immature chromatin or a fragmented DNA. Results from at least two, preferable three, separate seminal analyses must be 
obtained before a definitive conclusion can be drawn as wide biological variability exists within the same individual. Routine seminal analysis should include: a) physical characteristics of semen, including liquefaction, viscosity, $\mathrm{pH}$, color and odor; b) specimen volume; c) sperm count; d) sperm motility and progression; e) sperm morphology; f) leukocyte quantification; and g) fructose detection in cases where no spermatozoa is found and ejaculate volume is low. The criteria used for normality according to the World Health Organization (WHO) have been recently updated, as shown in Table 1 [2]. Approximately 2,000 men from eight countries whose partners had a time-to-pregnancy of $\leq 12$ months were chosen to provide reference distributions for semen parameters. One-sided lower reference limits (the fifth centile) were generated and have been proposed to be considered the lower cut-off limits for normality. Apart from total sperm number per ejaculate, the lower limits of these distributions are lower than that of previously presented 'normal' or 'reference' values [3-5] but are in agreement with recent observations [6-8].

The morphometric description of spermatozoa according to the strict criteria, described by Kruger et al. [9], has been incorporated in the current version of the WHO manual. The low proportions of normal spermatozoa, as defined by those selected from the endocervical mucus, produced very low reference limits for the fertile population. With such method, low values of $3-5 \%$ normal forms have been found to be optimal cut-off values to discriminate between fertile and infertile men whose spermatozoa were used for in vitro fertilization [10], intrauterine insemination [11] and spontaneous pregnancies [12]. Interpretation of the reference ranges requires an understanding that they provide a description of the semen characteristics of recent fathers. The reference limits should not be over-interpreted to distinguish fertile from infertile men accurately, but they do represent semen characteristics associated with a couple's achieving pregnancy within 12 months of unprotected sexual intercourse; as such, the limits provide only a standardized guide regarding a man's fertility. None of these values were able to solely distinguish fertile from infertile men, although morphology has been suggested to be of utmost importance [1012]. The coexistence of more than one altered seminal parameter significantly increases the risk for infertility [7].

\section{Genetic Testing for Y-Chromosome Infertility}

The long and short arms of Y-chromosome are respectively related to spermatogenesis and testicle development. The Y-chromosome region related to fertility is named azoospermia factor locus (AZF - azoospermia factor). Originally, three AZF regions have been defined: $\mathrm{AZFa}, \mathrm{AZFb}$, and $\mathrm{AZFc}$, which map on the long arm (Yq) in order from the centromere to the telomere. However, it has been demonstrated that $\mathrm{AZFb}$ and $\mathrm{AZFc}$ regions overlap [13]. A fourth region, named $\mathrm{AZFd}$, located between $\mathrm{AZFb}$ and AZFc has been also reported. These subregions contain multiple genes controlling different steps of spermatogenesis. The locus can present complete or partial microscopic deletions, isolated or in combination.

Clinically available molecular testing may reveal microdeletions in the long arm of the $\mathrm{Y}$ chromosome. The molecular diagnosis of $\mathrm{Y}$ chromosome deletion consists of a series of PCR (polymerase chain reaction) amplifications within relatively broad regions of the $\mathrm{Y}$ chromosome. Interstitial Yq microdeletions can remove various portions of the long arm, depending on breakpoints. Specific genes (i.e., USP9Y, DDX3Y, HSFY1, RBMY, CDY1, and DAZ) located along the $\mathrm{Y}$ chromosome should be included in the analysis to assess $\mathrm{Y}$ chromosome integrity [14]. The most common Y-chromosome deletion in infertile men is the one affecting the DAZ gene (deleted in azoospermia) located in $\mathrm{AZFc}$ region. The relative frequency of individual microdeletions are $60 \%, 5 \%$ and $16 \%$ for AZFc, AZFa and $\mathrm{AZFb}$ regions, but combined deletions occur in about $15 \%$ of the cases [15]. Since deletions tend to occur between large palindromic repeats, Repping et al. (2002) proposed a more appropriate nomenclature using the name of the flanking repeats for the types of recurrent deletions [13] (Fig. 1).

$\mathrm{Y}$ chromosome infertility is characterized by azoospermia or severe $\left(<1 \times 10^{6} \mathrm{sperm} / \mathrm{mL}\right.$ semen $)$ to moderate oligozoospermia (1-5 x $10^{6} \mathrm{sperm} / \mathrm{mL}$ semen $)$, but rare cases of mild oligozoospermia $\left(5-20 \times 10^{6} \mathrm{sperm} / \mathrm{mL}\right.$ semen $)$ may occur. Males with Y chromosome infertility usually have no obvious symptoms, although physical examination may reveal small testes and/or cryptorchidism or varicoceles. A single study suggested that one of the deletions of the $\mathrm{Y}$ chromosome in AZFc region (designated gr/gr) also causes a slight increase in susceptibility to testicular germ cell tumors [16]. The prevalence of $\mathrm{Y}$ chromosome microdeletions is estimated to be about 1:2000 to 1:3000 males. Overall, the frequency of $\mathrm{Yq}$ microdeletions in infertile males ranges between 5\%-12\%. Microdeletions can be found in $6 \%$ of men presenting with severe oligozoospermia and $15 \%$ of those with azoospermia [17]. For sperm counts between 1 and 5 million $/ \mathrm{mL}$ the detection rate drops down to $1.7 \%$ [18]. A marked variation in the reported worldwide frequency of AZF deletions among patients with nonobstructive (NOA) azoospermia from different regions of the world, i.e., $10-12 \%$ in USA, Australia and Asia, 2-10\% in Europe, and $7 \%$ in Brazil suggests that an ethnic variability may exist [14].

Pregnancies may be achieved by in vitro fertilization using intracytoplasmic sperm injection (ICSI) in males with Y-chromosome infertility exhibiting oligozoospermia or azoospermia with retrievable testicular sperm. It seems that the presence of $\mathrm{Yq}$ chromosome deletion has no apparent negative effect on fertilization and pregnancy rates, and it does not increase the risk for birth defects in children conceived via assisted reproduction $[18,19]$. The presence or absence of sperm (ejaculated or retrieved) in men with $\mathrm{Yq}$ microdeletions varies depending on the specific deletion. Detection of Y-chromosome microdeletions provides predictive information on the success of obtaining spermatozoa from the testicle of azoospermic individuals for ICSI. Testicular phenotypes (sperm counts and testicular histology) associated with microdeletions in the AZFa region are the most severe and include azoospermia and germ cell aplasia (Sertoli cell-only) pattern on histology while microdeletions in the AZFb subregion are associated with maturation arrest [20]. Testicular phenotypes associated with microdeletions restricted to $\mathrm{AZFc}$ ranged from azoospermia to moderate oligozoospermia whereas AZFd microdeletions manifest the broadest range of testicular phenotypes (from azoospermia to normal sperm counts). Large deletions, which involve multi- 


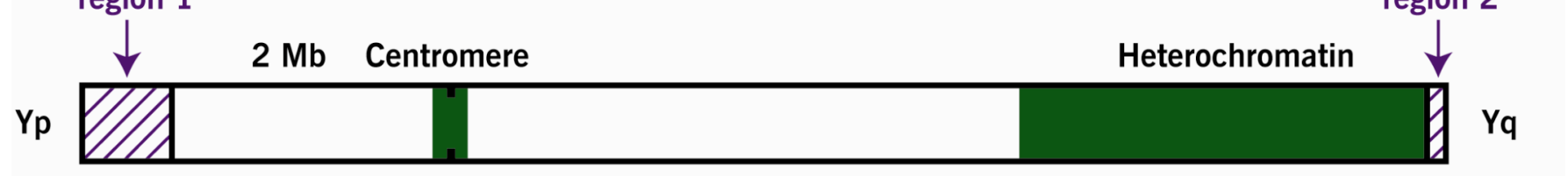

\section{Old Nomenclature $[\underset{A Z F a}{\mathbb{a}}$}

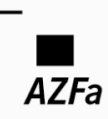

Current Nomenclature

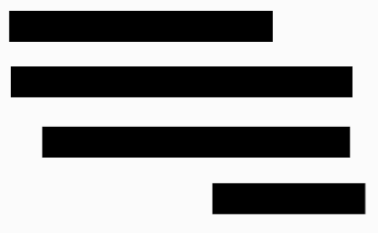

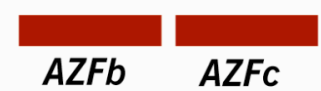

AZFC

Size

Fig. (1). Illustration of the Y chromosome in humans and the regions involved in fertility and infertility (Adapted from Am J Hum Genet 71(4), Repping S, et al., Recombination between Palindromes P5 and P1 on the Human Y Chromosome Causes Massive Deletions and Spermatogenic Failure, pages: 906-22, Copyright 2002, with permission from Elsevier). Interstitial or terminal deletions that include AZFa, often mediated by recombination between the HERV15yq1-HERV15yq2 repeats, usually produce the severe phenotype of Sertoli-cell-only syndrome. Interstitial or terminal deletions that include $\mathrm{AZFb}$ and/or $\mathrm{AZFb}+\mathrm{c}$ are mediated by recombination between palindromic repeats, either P5/proxP1, P5/distP1, or P4/distP1. These deletions usually result in azoospermia. Interstitial or terminal deletions that include AZFc only are mediated by recombination between the b2/b4 palindromic repeats and result in a variable phenotype, ranging from azoospermia and SCOS to severe or mild oligozoospermia. This type of deletion can occasionally be associated with normal fertility in younger males, with the phenotype worsening with age. Such individuals should consider cryopreservation of ejaculated sperm in early adulthood. Two partial deletions of AZFc, called b1/b3 and gr/gr, are considered polymorphisms.

ple AZF regions generally present with testicular phenotypes similar to those found in individuals with microdeletions restricted to AZFa [14]. In partial and complete AZFc deletion azoospermic patients, sperm can be retrieved from the testis in about $70 \%$ of the cases. In contrast, the chance of finding sperm in azoospermic men with complete AZFa or $\mathrm{AZFb}$ deletions is unlikely $[18,19]$.

$\mathrm{Y}$ chromosome infertility is inherited in a Y-linked manner. Because males with deletion of the AZF regions of the long arm of the $\mathrm{Y}$ chromosome are infertile, the deletions are usually de novo and therefore not present in the father of the proband. Rarely, within a family, the same deletion of the $\mathrm{Y}$ chromosome can cause infertility in some males but not in others; hence, some fertile males with deletion of the AZF regions have fathered sons who are infertile [21]. In pregnancies achieved from males with infertility caused by deletion of the AZF regions using ICSI, male offspring have the same deletion as their father, with a high risk of male infertility. Female fetuses from a father with a Y chromosome-deletion have no increased risk of congenital abnormalities or infertility. In pregnancies conceived via assisted reproduction and known to be at risk of resulting in a male with $\mathrm{Y}$ chromosome-deletion, specific prenatal testing or pre-implantation testing could be performed to determine the sex of the fetus and/or the presence of the $\mathrm{Y}$ chromosome-deletion.

\section{Sperm DNA Integrity Assays}

Sperm DNA fragmentation seems to be one of the most important causes of reduced fertility potential. Abnormalities in the male genome characterized by damaged sperm DNA may be indicative of male subfertility regardless of routine semen parameters which do not reveal DNA defects. An abnormal proportion of spermatozoa with fragmented DNA can be found in 5\% of infertile men with normal semen analyses and $25 \%$ of infertile men with abnormal semen analyses but is rarely seen in fertile men [15]. Advanced paternal age, inadequate diet intake, drug abuse, pesticide environmental exposure, tobacco use, varicocele, medical disease, hyperthermia, air pollution, genital inflammation or infectious diseases can be cited as possible causes, some of which are reversible. Fragmentation can be secondary to internal factors such as apoptosis and oxidative stress (a physiological mechanism secondary to a high concentration of free radicals), or external factors such as the presence of leukocytes.

Among the available methods used to assess sperm DNA integrity, the most common are TUNEL, Comet, Acridine Orange test and SCSA (Sperm Chromatin Structure Assay) [22]. TUNEL technique (Transferase-mediated dTUP nickend labeling) seems to be the most adequate as it offers the possibility to precisely identify all existing endogenous breaks in sperm DNA. It combines both enzymatic and immuno-hystochemical techniques for direct observation of DNA fragmentation using a fluorescence microscope or flow cytometry.

Sperm DNA damage levels are significantly different between fertile and infertile men, and therefore have important clinical significance [23]. The probability of fertilization in vivo and by intrauterine insemination (IUI) seems to be close to zero if the proportion of sperm cells with DNA damage exceeds $30 \%$ and $12 \%$, as detected 
respectively by SCSA or TUNEL [24, 25]. Sperm DNA damage is negatively correlated with embryo quality and blastocyst formation in in vitro fertilization (IVF), and with fertilization rates in both IVF and ICSI [26]. However, successful pregnancies in IVF/ICSI can be obtained using semen samples with a high proportion of DNA damage. Bungum et al. (2004) demonstrated that significantly higher clinical pregnancy rates $(52.9 \%$ vs. $22.2 \%)$ and delivery rates $(47.1 \% v s .22 .2 \%)$ were obtained after ICSI as compared to IVF when semen samples with high levels of sperm DNA damage were used, as previously suggested [27].

The activation of embryonic genome expression occurs at the four- to eight-cell stage in human embryos. The paternal genome may not be effective until that stage and it is speculated that elevated levels of sperm DNA strand breaks negatively impact the later stages of embryonic development. Aitken and Krausz (2001) suggested that sperm DNA damage is promutagenic and can give rise to mutations after fertilization, as the oocyte attempts to repair DNA damage prior to the initiation of the first cleavage. Mutations occurring at this point will be fixed in the germline but may be responsible for the induction of infertility, childhood cancer in the offspring and higher risk of imprinting diseases [28]. So far, however, follow-up studies of children born after ICSI compared with children born after conventional IVF have not been conclusive regarding the risks of congenital malformations, imprinting diseases and health problems. In vitro fertilization, in general, is associated with multiple gestations and increased risk of congenital abnormalities (including hypospadias) [29]. ICSI, in particular, carries an increased risk of endocrine abnormalities, as well as epigenetic imprinting effects [29]. Although the absolute risk of any of these conditions remains low, current data is limited and study populations are heterogenic [29-32]. It is therefore recommended that well-defined groups of ICSI with ejaculated sperm, ICSI with epididymal sperm and ICSI with testicular sperm, and a control group of naturally conceived children are closely followed up.

\section{Oxidative Stress Measurement}

The oxidative stress-induced sperm damage has been suggested to be a significant contributing factor in $30-80 \%$ of all cases of male infertility [33]. Reactive oxygen species (ROS) generation of can be exacerbated by environmental, infectious and lifestyle conditions. For example, exposure to phthalate, which is found in plastics used in food packaging, as well as beauty products, has been shown to cause DNA damage in sperm, as well as impaired spermatogenesis [34]. Cadmium and lead have been shown to increase testicular oxidative stress; in fact, increased rates of infertility and miscarriage have been found in welders as well as in workers of paint and battery manufacturers [35]. The food preservative sulfur dioxide directly increases intratesticular oxidative stress and diesel exhaust particulates have been shown to activate leukocyte ROS production [36]. Exposure to cigarette smoke increases both seminal leukocyte concentrations and seminal ROS generation, and decrease seminal levels of the antioxidant enzyme superoxide dismutase. Smoking has been noted to decrease concentrations of the seminal plasma antioxidants Vitamin $\mathrm{C}$ and $\mathrm{E}$ thereby reducing the oxidant scavenging capacity of spermatozoa and seminal fluid [37].
Oxidative stress (OS) can be measured during the clinical evaluation of the infertile male using direct and indirect assays [22]. Indirect assays measure the net oxidative sum of the balance between ROS production and the intra- and extracellular antioxidants that scavenge ROS, by assessing oxidation of the sperm cell membrane. The most widely used assay measures malondialdehyde (MDA), one of the final products of sperm cell membrane lipid peroxidation. Quantification of sperm DNA damage has also been used as an indirect assay of intracellular ROS-induced oxidant injury, although it is unable to distinguish oxidant-induced DNA damage from non-oxidative mechanisms. One possible solution is to measure oxidized deoxynucleotide, 8-oxo-7,8,dihydro 2 'deoxyguanoside ( 8 -OHdG), the specific byproduct of oxidant-induced DNA damage [38]. The most common direct method of measuring seminal ROS is via chemiluminescence. Luminol or lucigen probes can be used for quantification of redox activities of spermatozoa. Quantification of oxidative stress using luminol can be performed using a luminometer, which is less expensive and easier to use compared to the instruments for the indirect assays. Furthermore, luminol chemiluminescence has well-established ranges for fertile and infertile populations, bringing clinical relevance to its use [37].

\section{WHAT IS NEW IN TREAMENT}

\section{Antioxidant Therapy}

Recent reports have focused on the therapeutic management of oxidative stress in male infertility. Most studies, however, demonstrate a suboptimal experimental design making effective interpretation difficult. When considering therapeutic measures to decrease oxidative stress, counseling toward lifestyle modifications must be considered. An important challenge to proper counseling is the paucity of evidence supporting objective improvement in infertility following lifestyle modification [22]. Varicoceles have been shown to increase oxidative stress levels in the testes as well as in the semen. Varicocelectomy, in the other hand, may decrease seminal oxidative stress, increase seminal concentrations of antioxidants and also improve sperm quality [39]. In recent years, interest has increased regarding the role of antioxidants and B-vitamins as modulators of fertility [4043]. The antioxidants a-tocopherol (Vitamin E), ascorbic acid (Vitamin C) and the retinoids (Vitamin A) are potent scavengers of ROS. Deficiencies in vitamin B cause elevated homocysteine concentrations and impair the remethylation cycle. Vitamin B is involved in the methylation of phospholipids, proteins, DNA and RNA, and in the synthesis and repair of DNA. These processes are essential for normal spermatogenesis; therefore, derangements in these pathways may be detrimental for reproduction. Many studies have investigated the role of these and other vitamins and antioxidants in improving sperm parameters. Wong et al. (2002) demonstrated, in a randomized and placebo-controlled trial, that a 6-month administration of folic acid $(5 \mathrm{mg})$ and zinc phosphate (66mg) increased sperm counts in subfertile men [40]. Boxmeer et al. (2009) reported for the first time that low folate concentrations in the seminal plasma are associated with higher susceptibility of sperm DNA damage 
[41]. This novel finding is in line with the role of folate in both DNA synthesis and DNA and protein methylation processes. Folate shortage increases DNA fragility due to the misincorporation of uracil instead of thymine. During normal repair processes, when the removal of the misincorporated uracil fails, double-strand breaks resulting in chromosome instability may occur. Greco et al. (2005), studying a large cohort of infertile men with $>15 \%$ DNA-fragmented spermatozoa treated with either 1 gram of Vitamin C and E daily or placebo for two months, demonstrated beneficial effects of antioxidants in reducing the percentage of DNAfragmented spermatozoa; however, no effects were observed on the sperm parameters [42]. The authors further went on by demonstrating that supplementation with vitamins $\mathrm{E}$ and $\mathrm{C}$ significantly increased the rates of clinical pregnancy and implantation following ICSI, although a dose-response association was not seen between DNA fragmentation index (\% DFI) results and antioxidant intake [43]. Recently, a case series study involving couples with history of recurrent embryo losses suggested that an increased intake of antioxidantrich food or antioxidant supplements by men with high levels of sperm DNA fragmentation or lipid peroxidation may optimize gestational outcomes [44].

\section{Microsurgical Repair of Clinical Varicocele}

Several studies have demonstrated that the surgical treatment of clinical varicoceles is highly effective to decrease seminal oxidative stress, to increase seminal concentrations of antioxidants and to improve sperm quality [39, 45-47]. However, a meta-analysis of seven randomized, controlled trials of varicocele repair for male subfertility concluded that treatment of varicocele in men from couples with otherwise unexplained subfertility could not be recommended [48]. The study by Evers and Collins [48] was challenged by Ficcara et al. (2006), who argued that less than half of the studies of this Cochrane meta-analysis included patients with abnormal semen analysis and palpable varicocele. Moreover, Ficcara and colleagues [49] demonstrated that the methodology and statistical power of the Cochrane review were poor, thereby minimizing the significance of the conclusions against varicocele repair. The most recent meta-analysis on varicocelectomy by Marmar and colleagues (2007) unequivocally demonstrated the benefit of the surgical treatment of clinical varicoceles in infertile men with abnormal semen analyses [50]. The authors showed that the chances of obtaining a spontaneous conception were 2.8 times higher in

\section{Microsurgical Subinguinal Varicocele Repair}
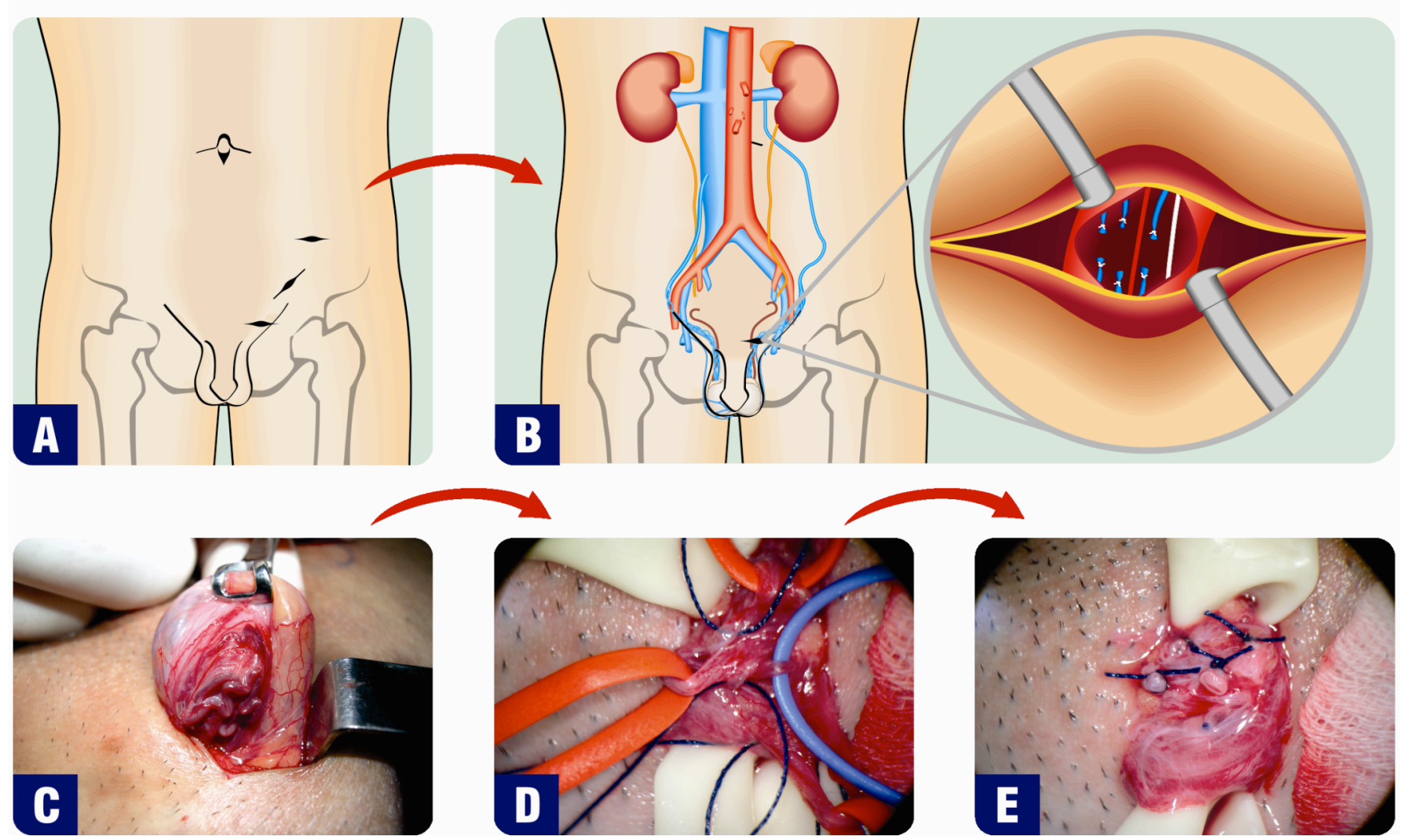

Fig. (2). Microsurgical Subinguinal Varicocele Repair. A) Incision sites commonly used for subinguinal, inguinal and retroperitoneal varicocele repair. B) In the subinguinal approach, a transverse incision is made just below the level of the external inguinal ring. $\mathrm{C}$ to $\mathrm{E}$ ) Intraoperative photographs of the spermatic cord. C) Dilated cremasteric veins are identified by elevating the spermatic cord with a Babcock clamp. D) Testicular artery (blue vessel loop), lymphatics (blue cotton suture) and dilated varicose veins (red vessel loops) are demonstrated. E) Final surgical aspect of varicose veins transected and ligated with non-absorbable sutures. 
the varicocelectomy group as compared to the group of patients who received either no treatment or medication. Recently, it has been shown that microsurgical varicocelectomy may also improve the outcomes of assisted reproduction in couples with varicocele-related infertility. Esteves et al. (2010) studied 242 infertile men with treated and untreated clinical varicoceles who underwent ICSI. The authors observed significantly higher pregnancy rates after ICSI in the group of men who underwent microsurgical varicocele repair before assisted conception. The clinical pregnancy $(60.0 \%$ vs. $45.0 \%)$ and live birth $(46.2 \%$ vs. $31.4 \%)$ rates after ICSI were significantly higher in the treated compared to the untreated group. The chances of achieving clinical pregnancy (odds-ratio [OR]: 1.82; 95\% confidence interval [CI]: 1.06-3.15) and live birth (OR: 1.87, 95\% CI: 1.08-3.25) by ICSI were significantly increased while the chance of miscarriage was reduced (OR: $0.433,95 \% \mathrm{CI}: 0.22-0.84$ ) after varicocele repair [51]. Inci et al. (2009) reported that varicocele repair prior to sperm retrieval significantly increased the chance of obtaining testicular sperm using microdissection testicular sperm extraction (micro-TESE) in a group of men with non-obstructive azoospermia (NOA) and clinical varicoceles. In their retrospective study, sperm retrieval rates were $53 \%$ and $30 \%$ in the treated and untreated men, respectively (OR: $2.63 ; 95 \%$ CI: 1.05-6.60) [52].

Currently, varicoceles are treated either by surgery (open with or without magnification and laparoscopy) or percutaneous embolization of the internal spermatic vein. Although techniques vary, the main concept is the occlusion of the dilated veins of the pampiniform plexus. The high retroperitoneal (Palomo), radiologic, and laparoscopic approaches are performed for internal spermatic vein ligation. The inguinal (Ivanissevich) and subinguinal approaches also allow the ligation of the external spermatic and cremasteric veins that may contribute to the varicocele. A recent systematic review including 4,473 infertile individuals was performed to define the best treatment modality for varicocele [53]. The authors concluded that open microsurgical inguinal or subinguinal varicocelectomy techniques resulted in higher spontaneous pregnancy rates and fewer recurrences and postoperative complications than laparoscopic, radiologic embolization and macroscopic inguinal or retroperitoneal varicocelectomy techniques (Fig. 2).

\section{Sperm Retrieval: Predictive Factors and Techniques}

The concept that elevated FSH levels are associated with male sterility has been challenged [54]. Serum FSH is an indirect reflection of the global (spermatogenic) function and histology of the testis. In cases of diffuse maturation arrest, for instance, adequate control feedback from germ cells and Sertoli cells exists despite the absence of sperm production; as a result, normal FSH levels are observed. Sperm retrieval techniques are able to obtain sperm from the testicle even in the cases of elevated serum FSH. However, it seems that the technique used for sperm retrieval plays a major role. Bromage et al. (2007) reported that the probability of testicular sperm retrieval using random TESE is decreased in NOA men with elevated FSH levels [55]. Conversely, Ramasamy et al. (2009) demonstrated that retrieval rates were nearly identical ( $\sim 60 \%$ success rate) regardless of FSH levels by using micro-TESE; these authors showed that preoperative $\mathrm{FSH}$ level of $\geq 15 \mathrm{IU} / \mathrm{L}$ resulted in greater odds of finding sperm with micro-TESE compared with normal FSH values [56]. Since its first description by Schlegel in 1998, microdissection testicular sperm extraction (microTESE) has become a recognized procedure for men with NOA [57]. Micro-TESE is more successful in sperm retrieval compared to single or multiple random biopsies in such cases [58]. Additionally, micro-TESE is associated with less effect on testicular function because it spares vessels during dissection and removes less tissue than random biopsies [59] (Fig. 3).

To date, there are no absolute predictors of successful sperm retrieval in men with NOA. Even the combination of the results of testicular histopathology and FSH levels provides only a "fair" prediction model (accuracy: 0.74) [60]. Nonetheless, Esteves et al. (2006) demonstrated that testicular histopathology in predictive of the chances of retrieving testicular sperm using micro-TESE. Sperm retrieval rates were significantly higher in the cases of hypospermatogenesis or maturation arrest $(100 \%$ and $75 \%$, respectively) as compared to Sertoli-cell only (32\%) [61]. These findings also highlight the concept that even the more adverse pattern on testicular histology does not determine if sperm are present elsewhere in the testis. However, it is still controversial whether invasive examination such as testicular biopsy should be performed, because it may cause inflammatory changes, hematoma, parenchymal fibrosis, or permanent devascularization of the testes.

Recently, the importance of surgical and medical treatment prior to sperm retrieval in NOA men has been highlighted. As already mentioned, treatment of clinical varicoceles prior to sperm retrieval may increase the chance of obtaining testicular sperm using micro-TESE in NOA men with clinical varicoceles [52]. Ramasamy and colleagues (2009) showed that men with Klinefelter syndrome (KS) with either normal or low baseline testosterone who responded to medical therapy had a better chance of sperm retrieval. In their study, men with serum testosterone levels less than $300 \mathrm{ng} / \mathrm{dL}$ received medical therapy (aromatase inhibitors, clomiphene or human chorionic gonadotropin) prior to micro-TESE. Patients who required medical therapy and responded to that treatment with a resultant testosterone of $250 \mathrm{ng} / \mathrm{dL}$ or higher had higher SR rate than men in whom post-treatment testosterone was less than $250 \mathrm{ng} / \mathrm{dL}$ (77\% vs. $55 \%$ ) [62]. Sperm retrieval rates were similar irrespective of the type of preoperative hormonal therapy, but the best SR rates $(86 \%)$ were achieved in men with normal baseline testosterone levels. Klinefelter syndrome is a common condition among men with NOA, accounting for about $11 \%$ of the cases [63]. The sterility of $\mathrm{KS}$ is due to the high prevalence of azoospermia, present in $92 \%$ of $\mathrm{KS}$ men able to provide a semen sample with the remainder having a median of 0.1 million sperm $/ \mathrm{mL}$. Nevertheless, sperm are found in $50 \%$ of cases on testicular exploration and in the couples who undergo ICSI, pregnancy rates range from $30 \%$ to $50 \%$ [63]. In KS, the births of more than 60 children have been reported and $\sim 50$ had karyotype analysis, all of which were normal $[63,64]$. 


\section{Microsurgical Testicular Sperm Extraction}

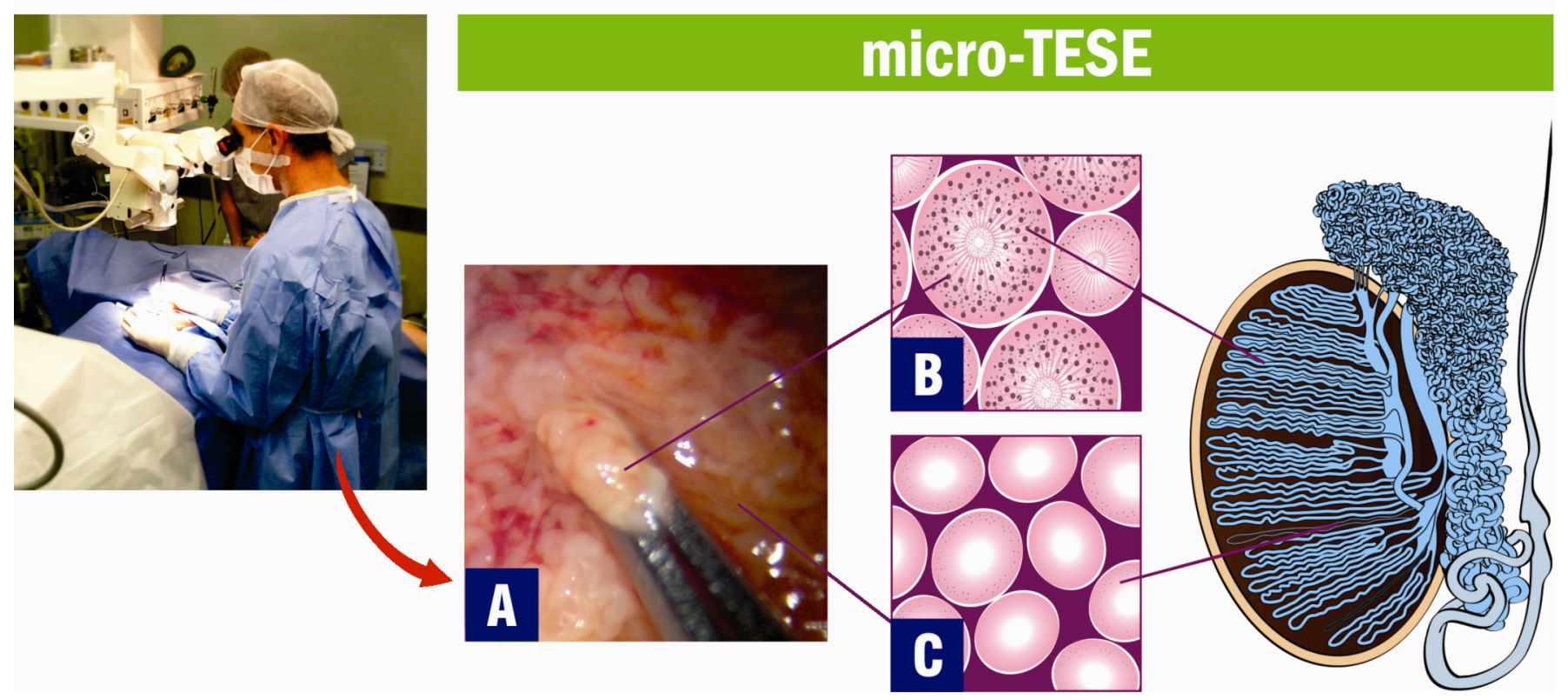

Fig. (3). Microsurgical testicular sperm extraction (micro-TESE). A) After the testicle is exteriorized, a single and large incision is made in an avascular area of the albuginea to expose the seminiferous tubules. B) Dilated tubules are identified and removed using the operating microscope and microsurgical technique (intraoperative photograph at x40-magnification). C) Illustration of the histopathology cross-section of a dilated seminiferous tubule with active spermatogenesis. D) Illustration of the histopathology cross-section of a thin tubule with Sertolicell only.

\section{EXPERT COMMENTARY}

Semen parameters within the reference interval do not guarantee fertility nor do values outside those limits necessarily imply male infertility or pathology. A man's semen characteristics need to be interpreted in conjunction with his clinical information. The reference limits provided by the WHO manual are from semen samples initiating natural conceptions; as such, they may indicate the need for infertility treatment but should not be used to determine the nature of that treatment.

Y-chromosome screening is indicated to infertile men presenting with moderate to severe (sperm count of $<5$ million $/ \mathrm{mL}$ ) oligozoospermia and NOA of unknown origin. It is debatable, however, if screening is valid when welldefined pathologies, such as radiation/chemotherapy or orchitis or trauma, are identified as the cause of oligo-/azoospermia. In other conditions, such as varicocele and cryptorchidism, $\mathrm{Yq}$ microdeletion screening is advisable since occasional cases of an association have been reported [65].

Sperm chromatin integrity, evaluated on neat semen, becomes particularly relevant when contact between the two gametes occurs in a more natural way and selective pressures operate to avoid the development of an embryo derived from sperm with a high load of genetic damage. ICSI can lead to pregnancy regardless of traditional sperm quality parameters and sperm chromatin structural integrity, but the potential adverse effects of using gametes with high loads of DNA damage are still unclear. Existing data justify the introduction of sperm DNA damage assessment to the male infertility workup, but evidence seems not to be strong enough to provide a clinical indication for routine use of these tests in infertility evaluation of men. Nonetheless, sperm DNA damage testing is being clinically utilized in at least four distinct clinical scenarios: i) to assess cases of unexplained infertility when traditional semen analysis falls into normal range and no evident female reproductive system pathologies can be revealed, ii) in selected cases of recurrent miscarriage, iii) to aid in the selection of the best assisted reproduction technology (ART) method, and iv) to define the sperm source, i.e. ejaculated or testicular sperm, for ICSI. However, the widespread use of sperm DNA testing and its inclusion as a routine testing into the clinical andrology laboratory is currently limited to the absence of robust standardized methods with rigorous quality control and high quality comparative clinical data. The methods used for detecting DNA damage differ in their mechanism of action and as a consequence what they ultimately reveal about the nature of that damage. Some of the assays are difficult to incorporate in most clinical andrology and ART laboratories due to equipment size/expense and hazardous reagents. Moreover, the ability to establish reliable positive and negative predictive values to be used for diagnosing fertility potential and ART outcome is still inadequate due to the lack of large, prospective adequately powered studies. Whether sperm DNA damage can be decreased by some treatment modalities, allowing these couples to switch from ICSI to IVF/IUI or even achieve a pregnancy in a natural way, remains to be elucidated. It is plausible to believe that administration of antioxidants, varicocelectomy and modifications in both the life-style and environmental exposure may play a role to infertile men with high sperm DNA damage. 
According to our experience involving approximately 200 individuals with NOA, successful SR rates of $55.7 \%$ have been achieved and results were similar in etiology categories of cryptorchidism, orchitis, genetic, radio-/chemotherapy and idiopathic [66]. Testicular histopathology results were predictive of sperm collection using both testicular sperm aspiration (TESA) and micro-TESE. Sperm retrieval by TESA was $100 \%$ and $82.3 \%$ in our group of NOA men presenting with either hypospermatogenesis or a history of previous successful TESA attempt. Using micro-TESE, SR rates were significantly higher than TESA in cases of SCO and maturation arrest (39.2\% vs. 22.8\%). Both methods yielded similar SR rates of $100 \%$ in cases of hypospermatogenesis. According to our data, the chances of retrieving spermatozoa and of achieving a live birth by ICSI are significantly increased in couples whose male partner had obstructive rather than non-obstructive azoospermia, thus indicating that the reproductive potential of infertile men undergoing ART is related to the type of azoospermia. [67].

\section{FIVE-YEAR REVIEW}

Y-chromosome infertility is associated with an intriguing AZF region which contains multiple genes, each of which is present in multiple copies. It is generally accepted that the chances of retrieving testicular spermatozoa in men presenting with complete deletions involving AZFa and/or $\mathrm{AZFb}$ regions is virtually zero. Deletion of the entire AZFb interval, for instance, has been associated with azoospermia arising from maturation arrest during meiosis. However, data are inconsistent with the phenotype typically seen in complete AZFb microdeletion [68, 69]. Longepied et al. (2010) described an exceptional case of one oligozoospermic man with a complete AZFb $+\mathrm{c}(\mathrm{P} 5 /$ distal-P1) deletion. Ploton et al. (2010) reported a case of an infertile man also presenting with oligozoospermia (3.6 million sperm $/ \mathrm{mL}$ ), small testes and slightly elevated FSH levels, and a microdeletion spanning a region between AZFb and AZFc. The authors showed that the microdeletion was transmissible over at least three generations. Genomic DNA from the patient's father gave the same pattern and during the course of investigation a pregnancy occurred in the patient's wife who delivered a healthy boy also showing the same microdeletion. In light of these recent observations, and since few patients with NOA and AZFa or AZFb deletions have undergone sperm retrieval, caution should be applied when counseling such individuals against testicular collection techniques.

Human sperm nuclei contain considerably fewer protamines than those of several mammalian species. Human sperm chromatin, therefore, is less regularly compacted and frequently contains DNA strand breaks. DNA fragmentation is characterized by both single (STB) and double DNA strand breaks (DSB), and is particularly frequent in the ejaculates of subfertile men [22]. Oocytes and early embryos may repair sperm DNA damage. Consequently, the biological effect of abnormal sperm chromatin structure depends on the combined effects of sperm chromatin damage and the capacity of the oocyte to repair it. Although the assessment of sperm DNA integrity seems to be an useful and potentially independent marker of fertility on both natural and assisted conception, recent reviews concluded that the clinical significance of sperm DNA integrity assessment remains unclear [70-72]. A relatively recent meta-analysis involving 22 relevant studies with infertile couples undergoing IVF and ICSI failed to support the concept that DNA integrity testing was more predictive for IVF than ICSI, and even that such testing is predictive of pregnancy outcome either in IVF or ICSI [70]. It is suggested that key objectives need to be urgently achieved for sperm DNA assessments to be widespread as effective clinical tools. These include: i) continuation of basic and translational research, ii) robust standardization of clinical assays and incorporation of systems for training staff and quality control; iii) high quality comparative clinical data and long-term follow-up of children resulting from assisted reproductive technology (ART) using sperm with defective sperm DNA damage [73, 74].

For men with NOA undergoing assisted conception treatments, follow-up of children born and identification of reliable predictors for successful sperm retrieval are of utmost importance. Recently, Sciurano et al. (2009) have shown that seminiferous tubuli with germ cells may be found in men with non-mosaic Klinefelter syndrome. Using fluorescence in situ hybridization (FISH) with probes for the Xcentromere and immunolocalization of meiotic proteins, they showed that all meiotic spermatocytes analyzed with FISH were euploid $(46, \mathrm{XY})$ and thus could form normal, haploid gametes. On the other hand, Sertoli cells showed two marks for the $\mathrm{X}$ chromosome, meaning that they were $47, \mathrm{XXY}$ [75]. These new findings may explain the high rate of normal children born after testicular sperm extraction plus ICSI when applied to Klinefelter patients. The authors speculated that these spermatogenic foci most probably originated from clones of spermatogonia that have randomly lost one of the $\mathrm{X}$ chromosomes, probably during periods of life when high spermatogonial mitotic activity occurred. Encouragingly, studies are now appearing addressing novel potential biomarkers for the prediction of sperm production within the testes. Magnetic resonance spectroscopy has been recently proposed as a possible tool to identify metabolic signatures associated with various histological states in infertile men. Based on ex-vivo analysis of testicular biopsy specimens, concentrations of several tissue metabolites were acquired and then reassessed in men with a diagnosis of NOA. A singular pattern could be determined for two testis histological states: normal and Sertoli cell-only (SCO) syndrome. Proliferating germ cells are related to high phospholipid synthesis and with elevated phosphocoline. Normal spermatogenesis spectroscopic pattern presented high peaks of phosphocoline as opposed to SCO. Further research in this area may aid in the identification of a distinct metabolic signature for sperm presence, regardless of testis histopathology [76].

\section{KEY ISSUES}

- World Health Organization established new reference values for human characteristics. Men whose partners had a time-to-pregnancy of $\leq 12$ months were chosen 
as individuals to provide reference distributions for semen parameters, as follows: semen volume of 1.5 $\mathrm{ml}$; sperm numbers of 39 and 15 million per ejaculate and per $\mathrm{mL}$, respectively; vitality of $58 \%$ live; progressive motility of $32 \%$ and morphologically normal forms (strict criteria) of $4.0 \%$. Semen parameters within the reference interval do not guarantee fertility nor do values outside those limits necessarily imply male infertility or pathology. A man's semen characteristics need to be interpreted in conjunction with his clinical information.

- $\quad$ About $10 \%$ of the infertile male population previously misdiagnosed as idiopathic have de novo Yq microdeletions. The presence or absence of sperm (ejaculated or retrieved) varies depending on the specific deletion. Y-chromosome screening is indicated to infertile men presenting with moderate to severe (sperm count of $<5$ million $/ \mathrm{mL}$ ) oligozoospermia and non-obstructive azoospermia of unknown origin or with associated conditions such as varicocele and cryptorchidism. Although no treatment exists, ICSI may be effective but the male offspring will inherit the same deletion as their father.

-

Assessment of sperm DNA integrity seems to be an useful and potentially independent marker of fertility on both natural and assisted conception. However, robust standardization of clinical assays and quality control as well as comparative clinical data are key elements that need to be urgently addressed for the widespread of sperm DNA assessments as effective clinical tools.

- $\quad$ Oxidative stress is associated with sperm quality and infertility. Therapeutic measures to decrease oxidative stress show promising results, and include lifestyle modifications, varicocelectomy in selected cases as well as use of vitamins and antioxidants. However, most studies still demonstrates a suboptimal experimental design, making effective interpretation difficult.

- Spontaneous conception is significantly increased after varicocele ligation in infertile men with clinical varicoceles and abnormal semen analyses. Recent data suggest that microsurgical treatment of clinical varicoceles optimize reproductive outcome of couples undergoing ICSI or micro-TESE sperm retrieval. The microsurgical approach is associated with lower recurrence and complication compared to conventional surgical modalities and embolization.

- In the subset of men with NOA, FSH levels are not predictive of successful sperm retrieval or sterility. Testicular microdissection sperm extraction yields the best SR rates for these patients, and success may be optimized by surgical treatment of clinical varicoceles or by medical therapy in non-mosaic KS men. In about $50 \%$ of non-mosaic Klinefelter syndrome azoospermic men testicular sperm are found by microTESE. Children born after testicular sperm extraction plus ICSI in KS have normal karyotype due to the fact that the minor foci of germ cells into the seminiferous tubuli are euploid.

\section{ACKNOWLEDGEMENTS}

None Declared.

\section{CONFLICT OF INTEREST}

None Declared.

\section{REFERENCES}

[1] Vital and Health Statistics, series 23, no.26, CDC. Available from: http://www.cdc.gov.[cited: $10^{\text {th }}$ Dec 2009].

[2] World Health Organization. WHO Laboratory Manual for the Examination and Processing of Human Semen, 5th ed. Geneva: WHO press 2010.

[3] World Health Organization. WHO Laboratory Manual for the Examination of Human Semen and Sperm-cervical Mucus Interaction, $2^{\text {nd }}$ ed. Cambridge: Cambridge University Press 1987.

[4] World Health Organization. WHO Laboratory Manual for the Examination of Human Semen and Sperm-cervical Mucus Interaction, $3^{\text {rd }}$ ed. Cambridge: Cambridge University Press 1992.

[5] World Health Organization. WHO Laboratory Manual for the Examination of Human Semen and Sperm-cervical Mucus Interaction, $4^{\text {th }}$ ed. Cambridge: Cambridge University Press 1999.

[6] Gunalp S, Onculoglu C, Gurgan T, Kruger TF, Lombard CJ. A study of semen parameters with emphasis on sperm morphology in a fertile population: an attempt to develop clinical thresholds. Hum Reprod 2001; 16(1): 110-4.

[7] Guzick DS, Overstreet JW, Factor-Litvak P, et al. Sperm morphology, motility, and concentration in fertile and infertile men. N Engl J Med 2001; 345(19): 1388-93.

[8] Menkveld R, Wong WY, Lombard CJ, et al. Semen parameters, including WHO and strict criteria morphology, in a fertile and subfertile population: an effort towards standardization of in-vivo thresholds. Hum Reprod 2001; 16(6): 1165-71.

[9] Kruger TF, Acosta AA, Simmons KF, Swanson RJ, Matta JF, Oehninger S. Predictive value of abnormal sperm morphology in vitro fertilization. Fertil Steril 1988; 49(1): 112-7.

[10] Coetzee K, Kruger TF, Lombard CJ. Predictive value of normal sperm morphology: a structured literature review. Hum Reprod Update 1998; 4(1): 73-82.

[11] Van Waart J, Kruger TF, Lombard CJ, Ombelet W. Predictive value of normal sperm morphology in intrauterine insemination (IUI): a structured literature review. Hum Reprod Update 2001; 7(5): 495500 .

[12] van der Merwe FH, Kruger TF, Oehninger SC, Lombard CJ. The use of semen parameters to identify the subfertile male in the general population. Gynecol Obstet Invest 2005; 59(2): 86-91.

[13] Repping S, Skaletsky H, Lange J. Recombination between palindromes P5 and P1 on the human Y chromosome causes massive deletions and spermatogenic failure. Am J Hum Genet 2002; 71(4): 906-22.

[14] Simoni M. Clinical consequences of microdeletions of the $\mathrm{Y}$ chromosome: the extended Münster experience. RBM Online 2008; 16(2): 289-30.

[15] Shefi S, Turek PJ. Definition and current evaluation of subfertile men. Int Braz J Urol 2006; 32(4): 385-97.

[16] Nathanson KL, Kanetsky PA, Hawes R, et al. The Y deletion gr/gr and susceptibility to testicular germ cell tumor. Am J Hum Genet 2005; 77(6): 1034-43.

[17] Foresta C, Moro E, Ferlin A. Y-chromosome microdeletions and alterations of spermatogenesis. Endocr Rev 2001; 22(2): 226-39.

[18] Stahl PJ, Masson P, Mielnik A, Marean MB, Schlegel PN, Paduch DA. A decade of experience emphasizes that testing for $\mathrm{Y}$ microdeletions is essential in American men with azoospermia and severe oligozoospermia. Fertil Steril 2010; 94(5): 1753-6.

[19] Oates RD, Silber S, Brown LG, Page DC. Clinical characterization of 42 oligospermic and azoospermic men with microdeletion of AZFc region of the $\mathrm{Y}$ chromosome, and of 18 children conceived with ICSI. Hum Reprod 2002; 17(11): 2813-24.

[20] Kamp C, Huellen K, Fernandes S, et al. High deletion frequency of the complete AZFa sequence in men with Sertoli-cell-only syndrome. Mol Hum Reprod 2001; 7(10): 987-94. 
[21] Saut N, Terriou P, Navarro A, Levy N, Mitchell MJ. The human Y chromosome genes BPY2, CDY1 and DAZ are not essential for sustained fertility. Mol Hum Reprod 2000; 6(9): 789-93.

[22] Kefer JC, Agarwal A, Sabanegh E. Role of antioxidants in the treatment of male infertility. Int J Urol 2009; 16(5): 449-457.

[23] Sharma RK, Sabanegh E, Mahfouz R, Gupta S, Thiyagarajan A, Agarwal A. TUNEL as a Test for Sperm DNA Damage in the Evaluation of Male Infertility. Urology 2010; 76(6): 1380-6.

[24] Bungum M, Humaidan P, Spano M, Jepson K, Bungum L, Giwercman A. The predictive value of sperm chromatin structure assay (SCSA) parameters for the outcome of intrauterine insemination, IVF and ICSI. Hum Reprod 2004; 19(6): 1401-8.

[25] Duran EH, Morshedi M, Taylor S, Oehninger S. Sperm DNA quality predicts intrauterine insemination outcome: a prospective cohort study. Hum Reprod 2002; 17(12): 3122-8.

[26] Seli E, Gardner DK, Schoolcraft WB, Moffatt O, Sakkas D. Extent of nuclear DNA damage in ejaculated spermatozoa impacts on blastocyst development after in vitro fertilization. Fertil Steril 2004; 82(2): 378-83.

[27] Larson-Cook KL, Brannian JD, Hansen KA, Kasperson KM, Aamold ET, Evenson DP. Relationship between the outcomes of assisted reproductive techniques and sperm DNA fragmentation as measured by the sperm chromatin structure assay. Fertil Steril 2003; 80(4): 895-902.

[28] Aitken RJ, Krausz C. Oxidative stress, DNA damage and the Y chromosome. Reproduction 2001; 122(4): 497-506.

[29] Alukal JP, Lamb DJ. Intracytoplasmic sperm injection (ICSI)what are the risks? Urol Clin North Am 2008; 35(2): 277-88.

[30] Knoester M, Helmerhorst FM, Vandenbroucke JP, et al. Artificial Reproductive Techniques Follow-up Project. Cognitive development of singletons born after intracytoplasmic sperm injection compared with in vitro fertilization and natural conception. Fertil Steril 2008; 90(2): 289-96.

[31] Belva F, Henriet S, Liebaers I, et al. Medical outcome of 8-year-old singleton ICSI children and a spontaneously conceived comparison group. Hum Reprod 2007; 22(2): 506-15.

[32] Woldringh GH, Besselink DE, Tillema AH, et al. Karyotyping, congenital anomalies and follow-up of children after intracytoplasmic sperm injection with non-ejaculated sperm: a systematic review. Hum Reprod Update 2010; 16(1): 12-9.

[33] Auger J, Eustache F, Andersen AG, et al. Sperm morphological defects related to environment, lifestyle and medical history of 1001 male partners of pregnant women from four European cities. Hum Reprod 2001; 16(12): 2710-7.

[34] Kasahara E, Sato EF, Miyoshi M, et al.Role of oxidative stress in germ cell apoptosis induced by di(2-ethylhexyl) phthalate. Biochem J 2002; 365(Pt 3): 849-56.

[35] Gennart JP, Buchet JP, Roels H, Ghyselen P, Cuelemans E, Lauerys R. Fertility of male workers exposed to cadmium, lead or manganese. Am J Epidemiol 1992; 135(11): 1208-19.

[36] Gonzalez-Flecha B. Oxidant mechanisms in response to ambient air particles. Mol Aspects Med 2004; 25(1,2): 169-82.

[37] Vine MF. Smoking and male reproduction: A review. Int J Androl 1996; 19(6): 323-7.

[38] Agarwal A, Makker K, Sharma R. Clinical relevance of oxidative stress in male factor infertility: an update. Am J Reprod Immunol 2008; 59(1): 2-11.

[39] Zini A, Blumenfeld A, Libman J, Willis J. Beneficial effect of microsurgical varicocelectomy on human sperm DNA integrity. Hum Reprod 2005; 20(4): 1018-21.

[40] WongWY, MerkusHM, ThomasCM, et al.Effects of folic acid and zinc sulfate on male factor subfertility: a double-blind, randomized, placebo-controlled trial. Fertil Steril 2002; 77(3): 491-8.

[41] Boxmeer JC, Smit M, Utomo E, et al. Low folate in seminal plasma is associated with increased sperm DNA damage. Fertil Steril 2009; 92(9): 548-56.

[42] Greco E, Iacobelli M, Rienzi L, Ubaldi F, Ferrero S, Tesarik J. Reduction of the incidence of sperm DNA fragmentation by oral antioxidant treatment. J Androl 2005; 26(3): 349-53.

[43] Greco E, Romano S, Iacobelli M, et al. ICSI in cases of sperm DNA damage: Beneficial effect of oral antioxidant treatment. Hum Reprod 2005; 20(9): 2590-4.

[44] Gil-Villa AM, Cardona-Maya W, Agarwal A, Sharma R, Cadavid A. Role of male factor in early recurrent embryo loss: do antioxidants have any effect. Fertil Steril 2009; 92(2): 565-71.
[45] Esteves SC, Glina S. Recovery of spermatogenesis after microsurgical subinguinal varicocele repair in azoospermic men based on testicular histology. Int Braz J Urol 2005; 31(6): 541-8.

[46] Agarwal A, Deepinder F, Cocuzza M, et al. Efficacy of varicocelectomy in improving semen parameters: new metaanalytical approach. Urology 2007; 70(3): 532-8.

[47] Smit M, Romijn JC, Wildhagen MF, et al. Decreased sperm DNA fragmentation after surgical varicocelectomy is associated with increased pregnancy rate. J Urol 2010; 183(1): 270-4.

[48] Evers JLH, Collins JA. Assessment of efficacy of varicocele repair for male subfertility: A systematic review. Lancet 2003; 361(9372): 1849-52.

[49] Ficcara V, Cerruto MA, Liguori G, et al.Treatment of varicocele in subfertile men: The Cochrane review - a contrary opinion. Eur Urol 2006; 49(2): 258-63.

[50] Marmar JL, Agarwal A, Prabaskan S, et al. Reassessing the value of varicocelectomy as a treatment for male subfertility with a new meta-analysis. Fertil Steril 2007; 88(3): 639-48.

[51] Esteves SC, Oliveira FV, Bertolla RP. Clinical outcome of intracytoplasmic sperm injection in infertile men with treated and untreated clinical varicocele. J Urol 2010; 184(4): 1442-6.

[52] Inci K, Hascicek M, Kara O, et al. Sperm retrieval and intracytoplasmic sperm injection in men with nonobstructive azoospermia, and treated and untreated varicocele. J Urol 2009; 182(4): 1500-5.

[53] Cayan S, Shavakhabov S, Kadioglu A. Treatment of palpable varicocele review in infertile men: a meta-analysis to define the best technique. J Androl 2009; 30(1): 33-40.

[54] Hung AJ, King P, Schlegel PN. Uniform testicular maturation arrest: a unique subset of men with nonobstructive azoospermia. J Urol 2007; 178(2):608-12.

[55] Bromage SJ, Falconer DA, Lieberman BA, Sangar V, Payne SR. Sperm retrieval rates in subgroups of primary azoospermic males. Eur Urol 2007; 51(2): 534-9.

[56] Ramasamy R, Lin K, Gosden LV, Rosenwaks Z, Palermo GD, Schlegel PN. High serum FSH levels in men with nonobstructive azoospermia do not affect success of microdissection testicular sperm extraction. Fertil Steril 2009; 92(2): 590-3.

[57] Schlegel PN. Testicular sperm extraction: Microdissection improves sperm yield with minimal tissue excision. Hum Reprod 1999; 14(1): 131-5.

[58] Okada H, Dobashi M, Yamazaki T, et al. Conventional versus microdissection testicular sperm extraction for nonobstructive azoospermia. J Urol 2002; 168(3): 1063-7.

[59] Ramasamy R, Yagan N, Schlegel PN. Structural and functional changes to the testis after conventional versus microdissection testicular sperm extraction. Urology 2005; 65(6): 1190-4.

[60] Tournaye H, Verheyen G, Nagy P, et al. Are there any predictive factors for successful testicular sperm recovery in azoospermic patients. Hum Reprod 1997; 12(1): 80-6.

[61] Esteves SC, Verza Jr S, Gomes AP. Successful retrieval of testicular spermatozoa by microdissection (micro-TESE) in nonobstructive azoospermia is related to testicular histology [abstract]. Fertil Steril 2006; 86(Suppl 1): 354.

[62] Ramasamy R, Ricci JA, Palermo GD, Gosden LV, Rosenwaks Z, Schlegel PN. Successful fertility treatment for Klinefelter's syndrome. J Urol 2009; 182(3): 1108-13.

[63] Schiff JD, Palermo GD, Veeck LL, Goldstein M, Rosenwaks Z, Schlegel PN. Success of testicular sperm injection and intracytoplasmic sperm injection in men with Klinefelter syndrome. $\mathrm{J}$ Clin Endocrinol Metab 2005; 90(11): 6263-7.

[64] Staessen C, Tournaye H, Van Assche E, et al. PGD in 47, XXY Klinefelter's syndrome patients. Hum Reprod Update 2003; 9(4): 319-30.

[65] Foresta C, Moro E, Ferlin A. Y Chromosome Microdeletions and Alterations of Spermatogenesis. Endocrine Rev 2001; 22 (2): 22639.

[66] Esteves SC, Verza S, Prudencio C, Seol B. Sperm retrieval rates (SRR) in nonobstructive azoospermia (NOA) are related to testicular histopathology results but not to the etiology of azoospermia [abstract]. Fertil Steril 2010; 94(Suppl 1): 132.

[67] Prudencio C, Seol B, Esteves SC. Reproductive potential of azoospermic men undergoing intracytoplasmic sperm injection is dependent on the type of azoospermia [abstract]. Fertil Steril 2010; 94 (Suppl 1): 232-3. 
[68] Longepied G, Saut N, Aknin-Seifer I, et al. Complete deletion of the AZFb interval from the Y chromosome in an oligozoospermic man. Hum Reprod 2010; published online: August 17, 2010.

[69] Plotton I, Ducros C, Pugeat M, Morel Y, Lejeune H. Transmissible microdeletion of the Y-chromosome encompassing two DAZ copies, four RBMY1 copies, and both PRY copies. Fertil Steril 2010; 94(7): 2770.

[70] Collins JA, Barnhart KT, Schlegel PN. Do sperm DNA integrity tests predict pregnancy with in vitro fertilization. Fertil Steril 2008; 89(4): 823-31.

[71] Zini A, Sigman M. Are tests of sperm DNA damage clinically useful. Pros and cons. J Androl 2009; 30(3): 219-29.

[72] Sakkas D, Alvarez JG. Sperm DNA fragmentation: mechanisms of origin, impact on reproductive outcome, and analysis. Fertil Steril 2010; 93(4): 1027-36.
[73] Barratt CL, Aitken RJ, Bj€orndahl L, Carrell DT, de Boer P, Kvist $\mathrm{U}$, et al. Sperm DNA: organisation, protection and vulnerability: from basic science to clinical applications - a position report. Hum Reprod 2010; 25(4): 824-38.

[74] Barratt CRL, De Jonge CJ. Clinical relevance of sperm DNA assessment: an update. Fertil Steril 2010; 94(6): 1958-9.

[75] Sciurano RB, Luna Hisano CV, Rahn MI, et al. Focal spermatogenesis originates in euploid germ cells in classical Klinefelter patients. Hum Reprod 2009; 24(9): 2353-60.

[76] Aaronson DS, Iman R, Walsh TJ, et al. A novel application of $1 \mathrm{H}$ magnetic resonance spectroscopy: non-invasive identification of spermatogenesis in men with non-obstructive azoospermia. Hum Reprod 2010; 25(4): 847-52.

Received: January 02, 2010

Revised: March 08, 2010

Accepted: July 26, 2010

(C) Esteves and Agarwal; Licensee Bentham Open.

This is an open access article licensed under the terms of the Creative Commons Attribution Non-Commercial License (http://creativecommons.org/licenses/by$\mathrm{nc} / 3.0 /$ ), which permits unrestricted, non-commercial use, distribution and reproduction in any medium, provided the work is properly cited. 